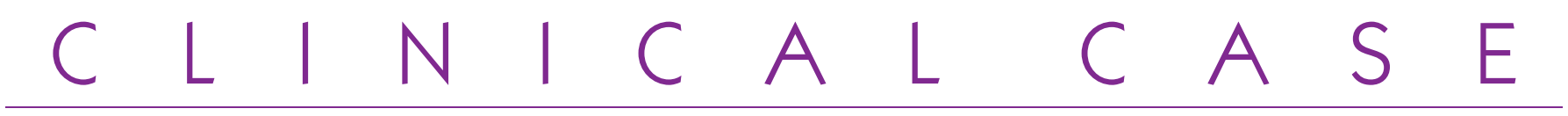

\title{
Treatment of Class II non-extraction using the Bioprogressive method
}

\section{P. Guezenec}

CD SQODF, membre titulaire de la SBR, pratique libérale à Saint-Brieuc

\section{INTRODUCTION}

Constance is a young patient who has a Class II with a significant overbite. The case is complicated by an impacted upper canine and a significant dento-maxillary discrepancy. The diagnosis and subsequent treatment plan may lead to the extraction of premolars but then the problem arises concerning the esthetic impact of these extractions.

\section{TREATED CASE}

Constance's first consultation was in December 2005 (9 years, 3 months) (Fig. 1), 11 primary teeth were present, 22 had not yet erupted. We decided to wait for eruption of 22. She returned in July 2008, aged 11 years, 9 months (Fig. 1)

Her reason for the consultation was the lower incisor crowding. During this consultation, the absence of 23 , the overbite and
We use the VTO (visual treatment objective) approach currently used in the Rickett's Bioprogressive Technique in order to evaluate the choice: to extract or not to extract. And also to next make a decision that conforms with our objectives even if the degree of difficulty of the treatment mechanics is increased. Our objective, dictated by the patient's soft tissues, corresponds to a "patient-centered" approach to treatment.

the upper midline deviation towards the left were noted. The file was completed in December 2008.

\section{Clinical exam}

The functional matrix is neutral, no functional problems were found. A dust allergy is noted.
Address for correspondence:

Patrick Guézénec

3, bd Waldeck Rousseau

22000 Saint-Brieuc

Odf.guezenec@wanadoo.fr
Article received: 21-03-2014. Accepted for publication: 25-04-2014. 


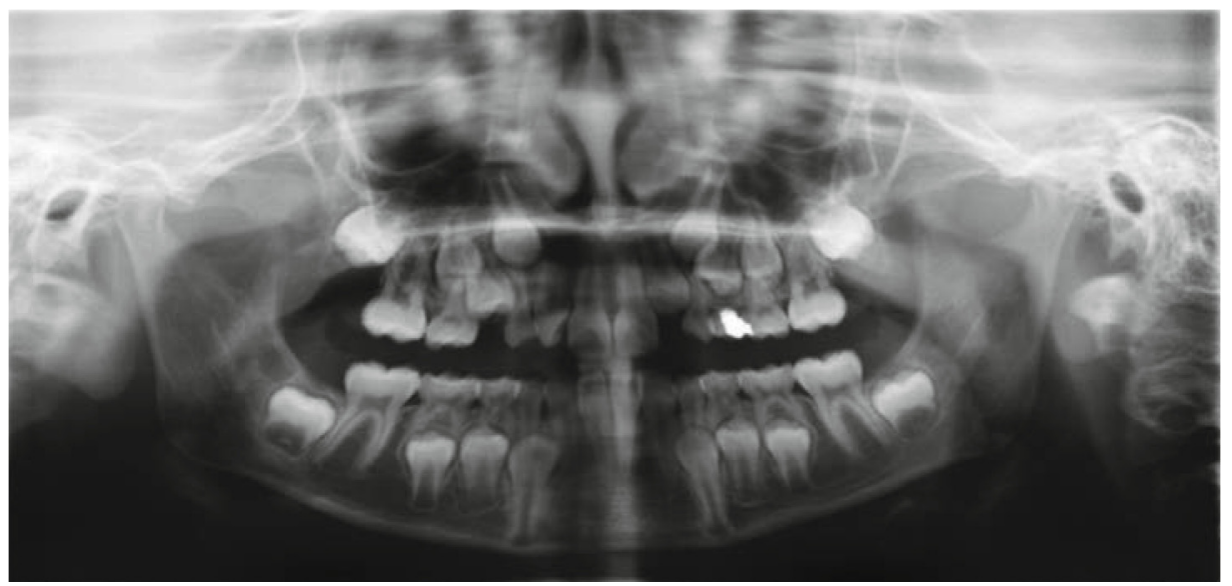

Figure 1

Panoramic radiograph 1st consultation 9 years 3 months.

The dental exam shows a bilateral half-step Class II, 23 is impacted.

The overjet measures $3 \mathrm{~mm}$ with a $90 \%$ overbite. The dento-maxillary discrepancy measured $9 \mathrm{~mm}$. The lower inter-canine width is $25 \mathrm{~mm}$. All the permanent teeth have erupted except 17, 23, 27. As well as the wisdom teeth (Fig. 2a to c, Fig. 3a, b).

The panoramic shows the mandibular third molars very tilted forward (Fig. 4).

The maxilla is " $V$ " shaped with a closed off (locked) mandible in the transverse and sagittal directions. The upper midline is deviated $3 \mathrm{~mm}$ to the left.

\section{- Esthetic evaluation}

The profile is retrusive, the lips are behind the esthetic line. The upper lip measures $18 \mathrm{~mm}$, the upper incisor is found in repose at $6 \mathrm{~mm}$ and in smiling, Constance exposes $8 \mathrm{~mm}$ of her central incisor. There is no gingiva visible when she smiles (Fig. 5a to c).

\section{- Evaluation of the TMJ}

Maximum opening: $42 \mathrm{~mm}$

Maximum propulsion: $9 \mathrm{~mm}$

Right lateral excursion: $9 \mathrm{~mm}$

Left lateral excursion: $9 \mathrm{~mm}$ (in consideration of the midline deviation)

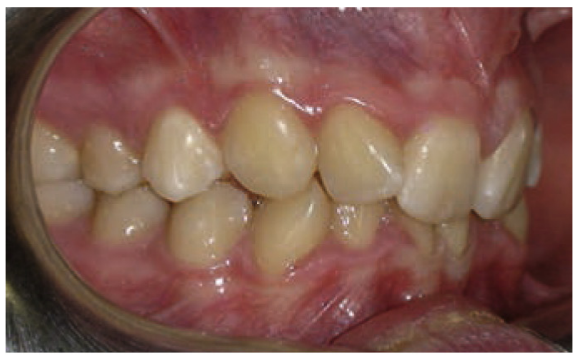

a

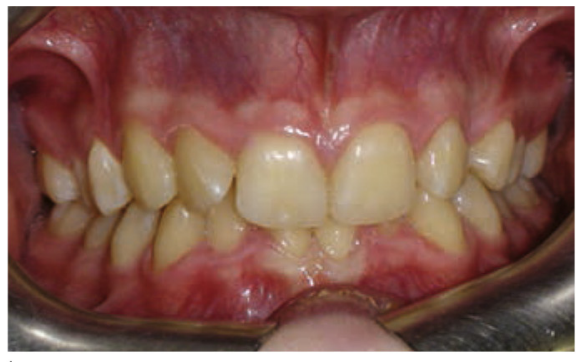

$\mathrm{b}$

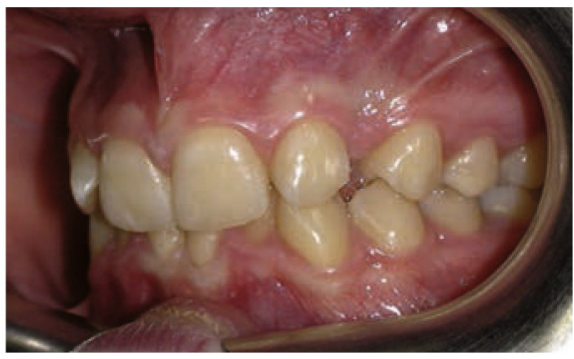

Figure 2

$a, b$ and $c$ : Intraoral views at the start of treatment (11 years 9 months). 


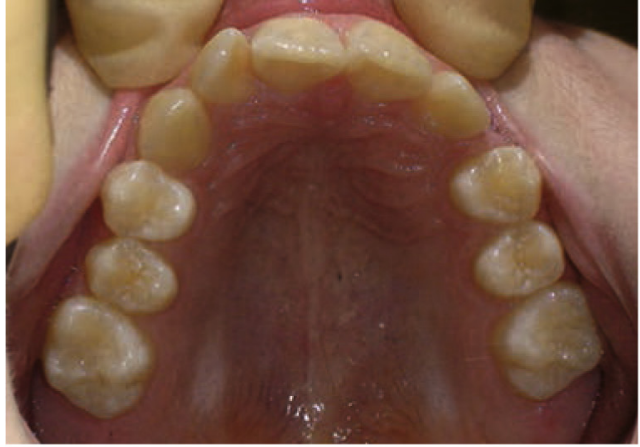

a

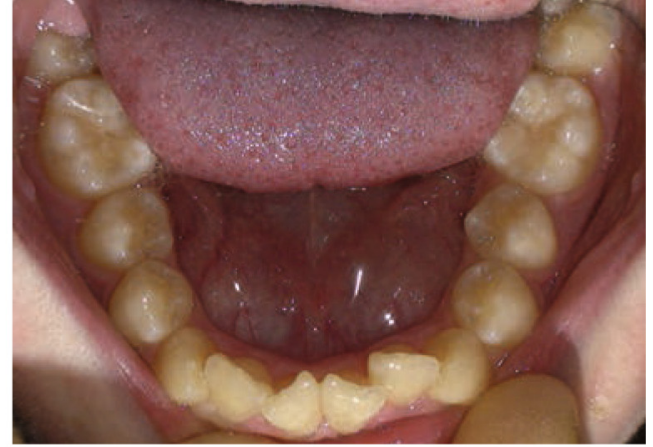

b

Figure 3

$a$ and b: Maxillary and mandibular arches at the start (11 years, 9 months).

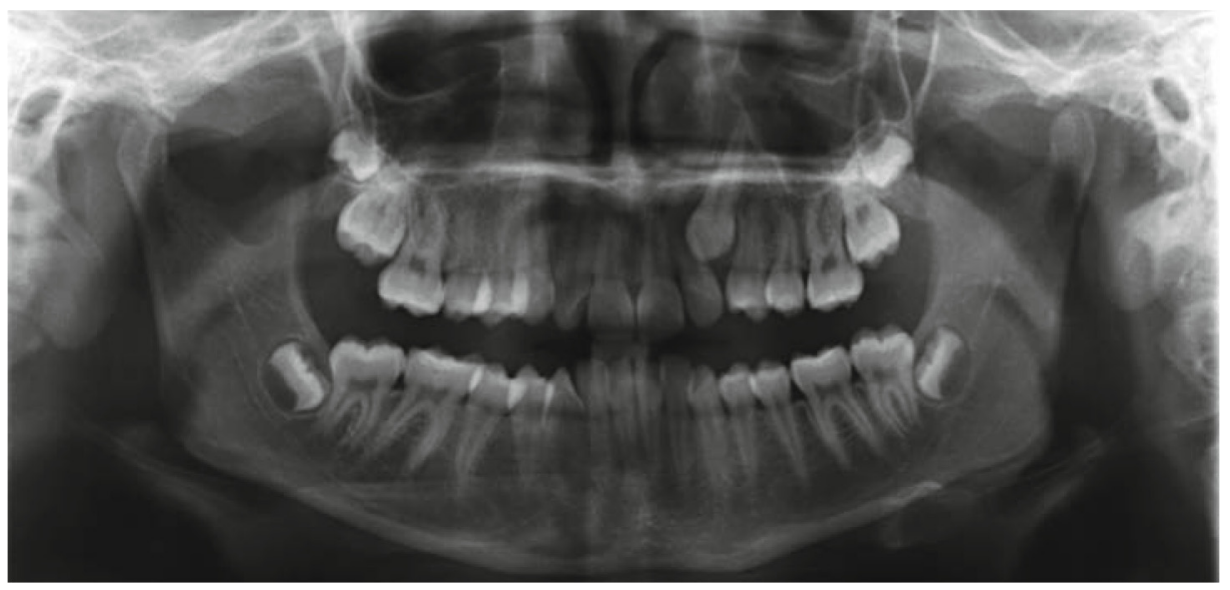

Figure 4

Panoramic radiograph at the start of treatment (11 years, 9 months).

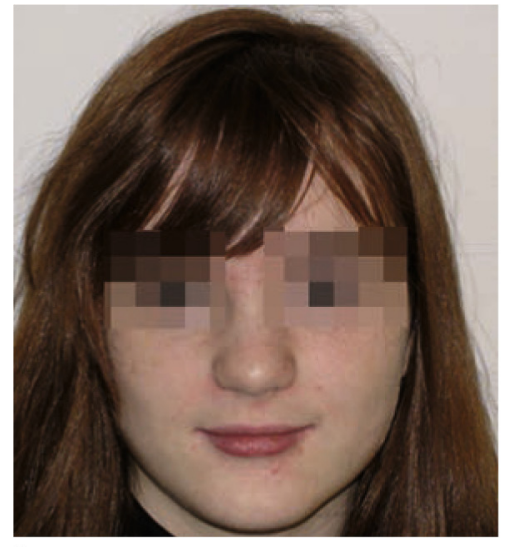

a

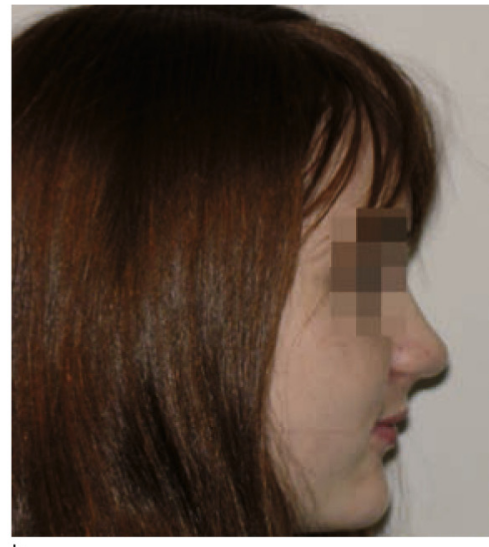

b

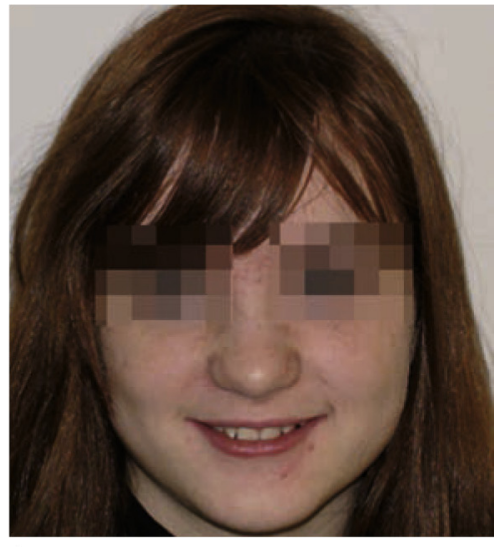

$\mathrm{C}$

น

Figure 5

a) Start treatment frontal facial view; b) start treatment profile view; c) start treatment smiling view. 


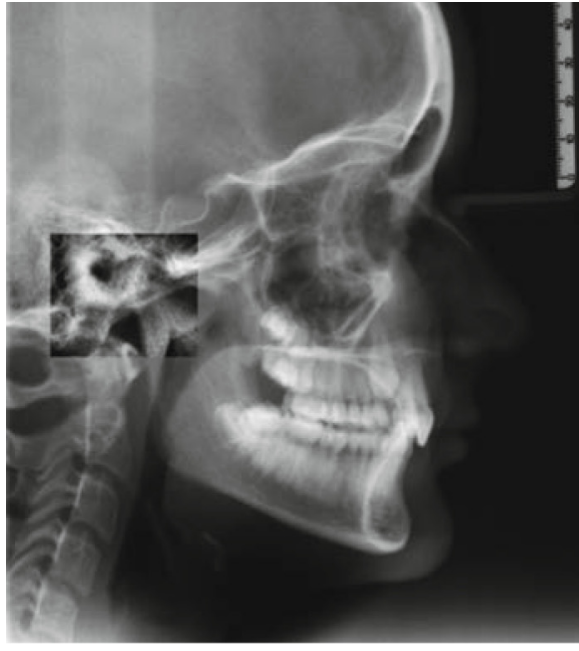

a

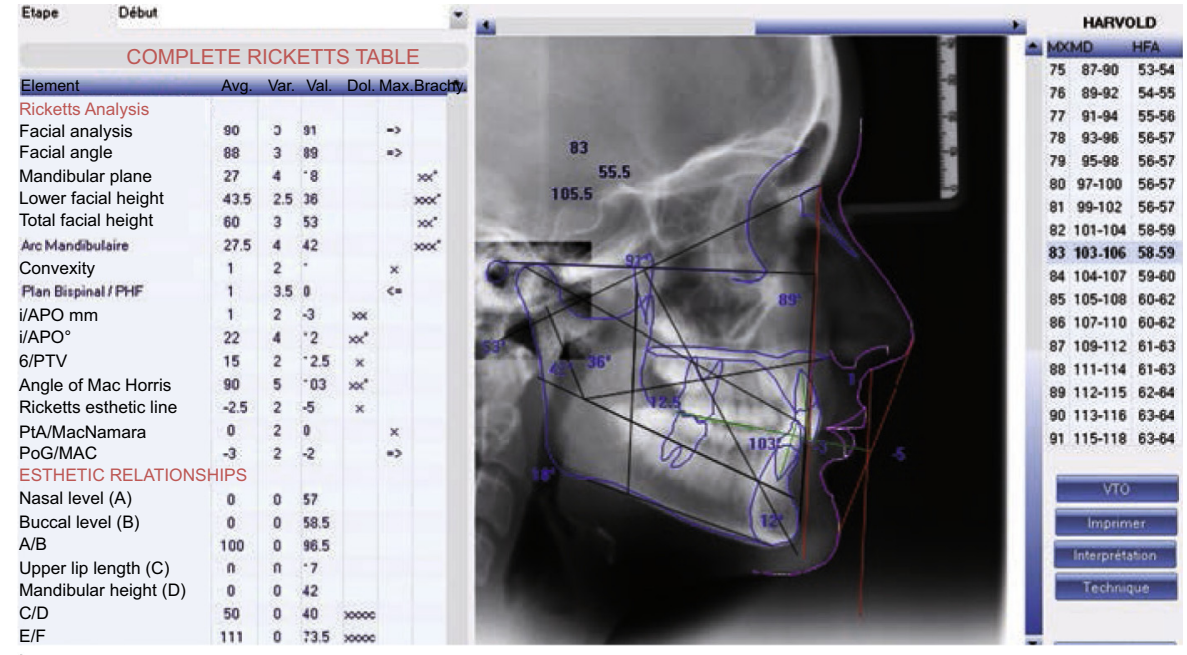

Figure 6

a and b: Brachyfacial growth, skeletal deep bite, skeletal Class I.

a) Start treatment lateral profile teleradiograph; b) start treatment Ricketts profile cephalometic analysis.

These movements do not indicate any problems with the TMJ, no pain, no cracking (clicking).

\section{- Psychological evaluation}

Constance is highly motivated. Constance's oral hygiene is very good.

\section{Clinical degree of difficulty is level 3}

The treatment plan report was done in January 2009, with specifications and informed consent.

\section{Cephalometic analyses (Fig. 6a, b)}

The triangle of Harvold shows normal proportions $(83 / 105 / 55)$ with a decrease in the height of the lower one-third of the face.

The upper incisors are in linguoversion blocking the lower incisors.

The Grummons facial analysis indicates the need to control the transverse dimension, slightly deficient, and the deviation of the upper midline from alignment with the median axis of the face (Fig. 7a, b).

The VTO done without extractions in order to avoid retrusion of the lips could possibly lead to a disastrous profile. The objectives are thus to control the transverse direction of both arches, to correct the Class II molar by retracting 16 and 26 followed by 13 , to correct the median (midline) superior point and open the space for 23. The upper and lower incisors can be torqued to increase the arch length considering the brachyfacial character of the skeletal pattern (Fig. 8a, b).

\section{Degree of difficulty of the objectives of level 3}

The option of extracting 4 premolars is excluded for the esthetic reasons mentioned above even though, without extractions of premolars, the degree of difficulty of the mechanics is more difficult. 

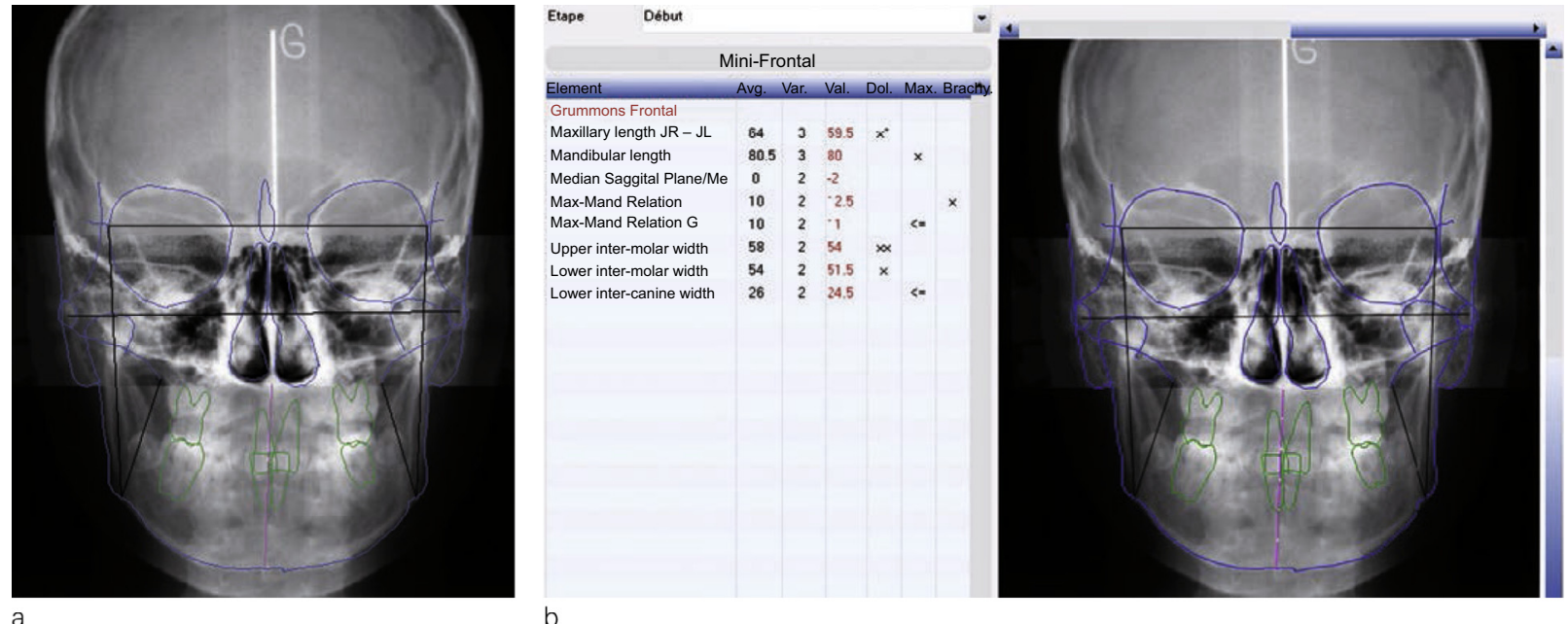

Figure 7

a) Start treatment facial teleradiograph; b) start treatment Ricketts frontal analysis.
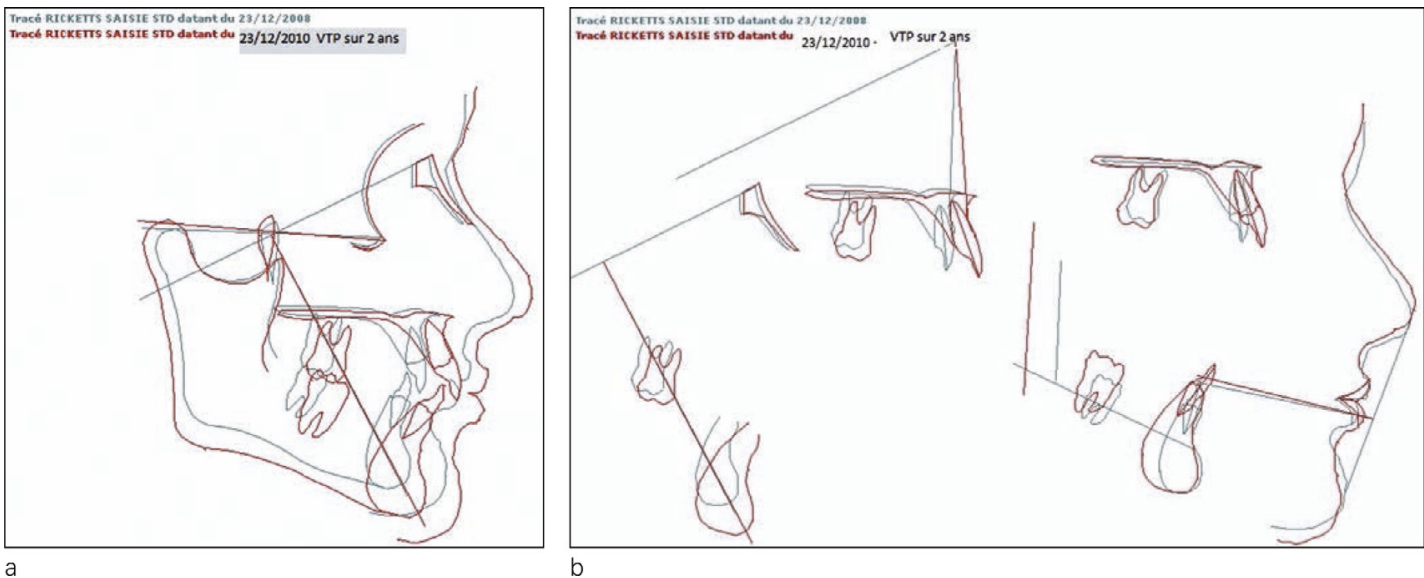

Figure 8

a) VTO for planning over 2 years; b) VTO - 5 Ricketts superimpositions.

\section{Treatment plan}

Hilgers pendulum for retraction of 16 and 26 and transverse directional control

Molar anchorage using an upper base arch, lateral sectionals, opening of space for 23 .

Lower advance arch, Class II intermaxillary traction on 13 and 24.
Alignment into the arch for 23 (unimpaction planned) extraction of the wisdom teeth.

Finishing, retention.

\section{- Treatment progress}

19.02.09: impression for fabrication of the pendulum, placed on 18.03.09 (Fig. 9a to c, Fig. 18a, b). 


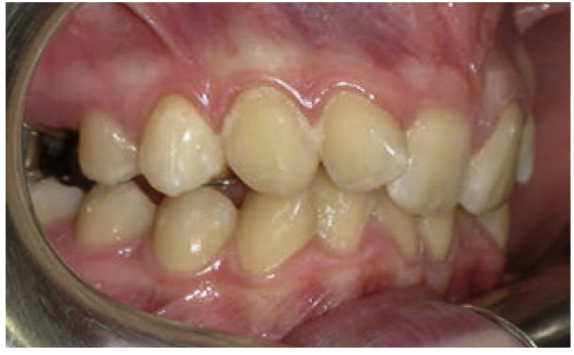

a

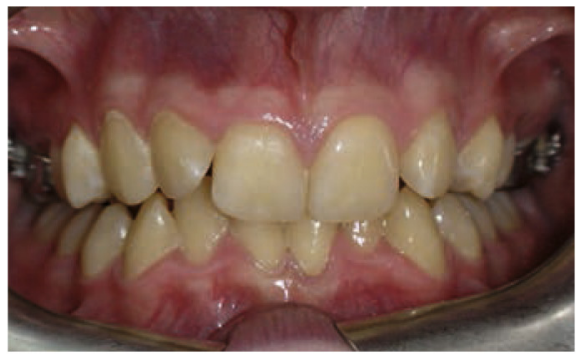

b

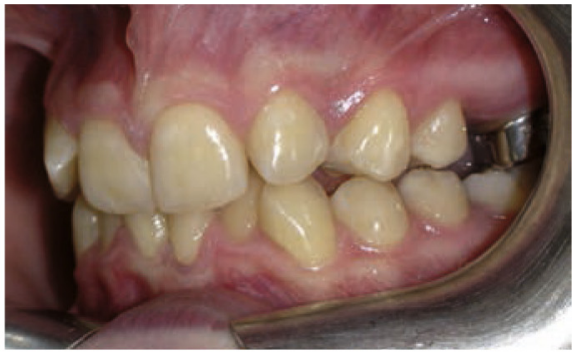

Figure 9

$a, b$, and $c$ : Intraorals after 6 months.

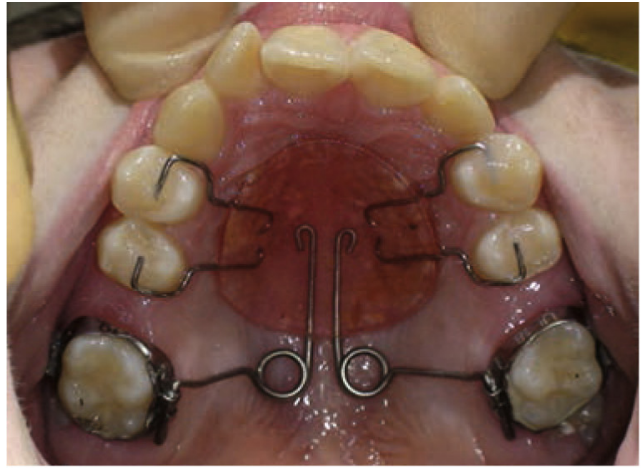

a

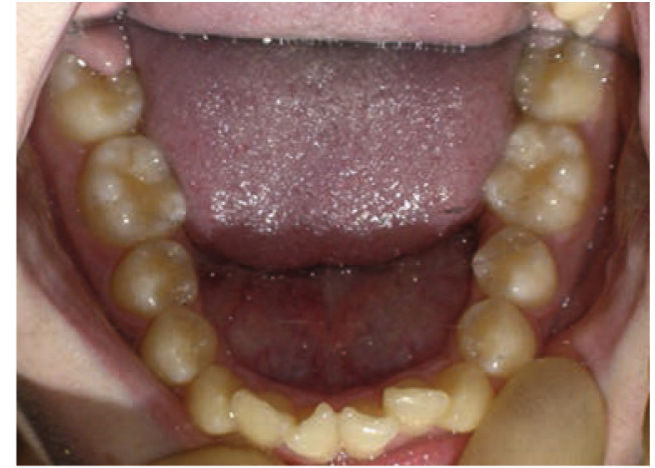

b

Figure 10

a) Hilgers pendulum after 6 months; b) mandibular arch after 6 months.

03.11.09: bonding of maxillary arch, retraction of 1525 , maxillary base arch Elgiloy $16 \times 22$ with and /016 arch overlay, sectional from 36 46, banding of lower arch on 17.02.10, lowewr advance arch in Elgiloy $16^{2}$. Intrusion of the upper incisors. Control of transverse direction with the pendulum then with expansion of the base arches (Fig. 11a to c, Fig. 12a, b).

30.07.10 bonding of lingual buttons on 2425 to correct rotation of the premolars.

04.11.10: request for exposure of 23 and extraction of the wisdom teeth.
13.01.11: Class II intermaxillary traction (fox* elastics $1 / 43.5$ oz worn 24 hrs/day).

10.02.11: bonding 23 (Fig. 14a to $\mathrm{c}$, Fig. 15a, b).

26.05.11: segmentation

23.06.11: stop intermaxillary traction, Finishing and stabilization.

01.09.11: upper removal and impression for retention by thermoformed appliance, placed on 15.09.11.

01.12.11: lower removal and bonded lower retention from 33 to 43 (Fig. 16a to C, Fig. 17a, b, Fig. 18a to $\mathrm{C})$. 


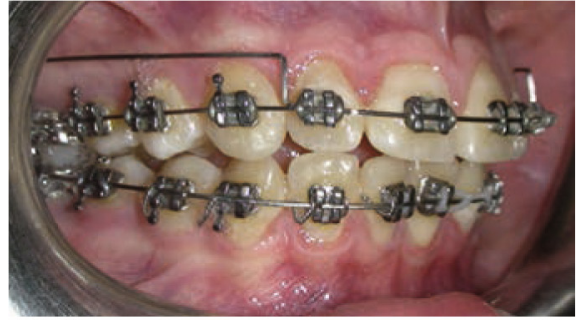

a

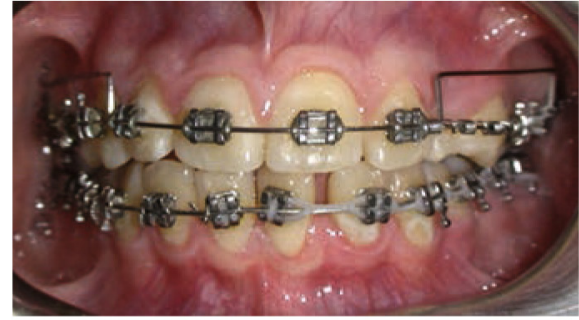

$\mathrm{b}$

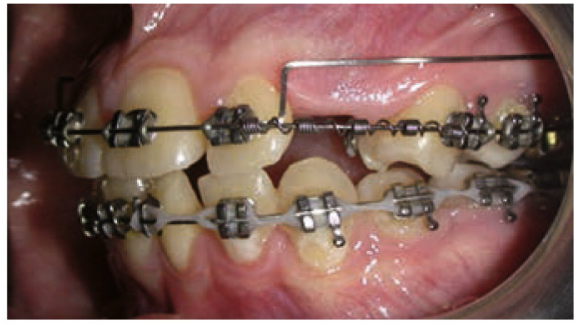

C

Figure 11

$a, b$ and $c$ : Intraorals after 12 months.

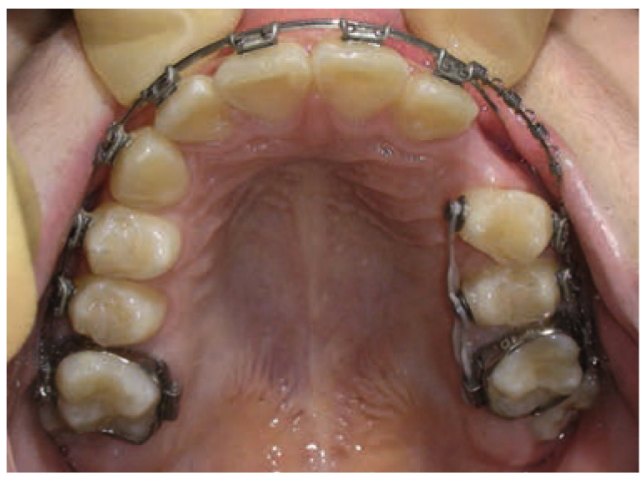

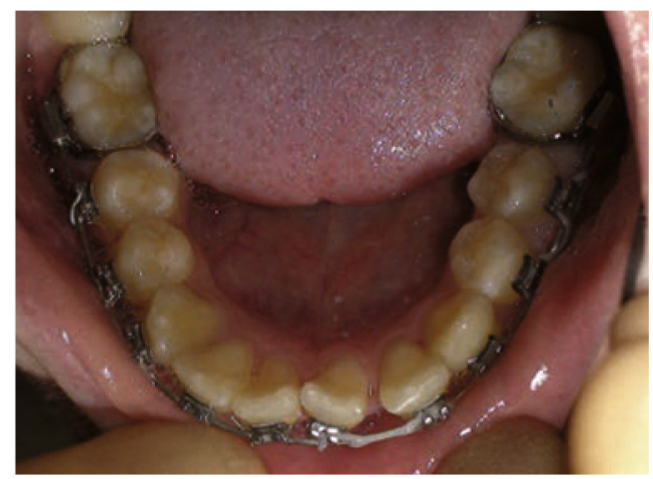

b

Figure 12

$a$ and b: Maxillary and mandibular arches after 12 months.

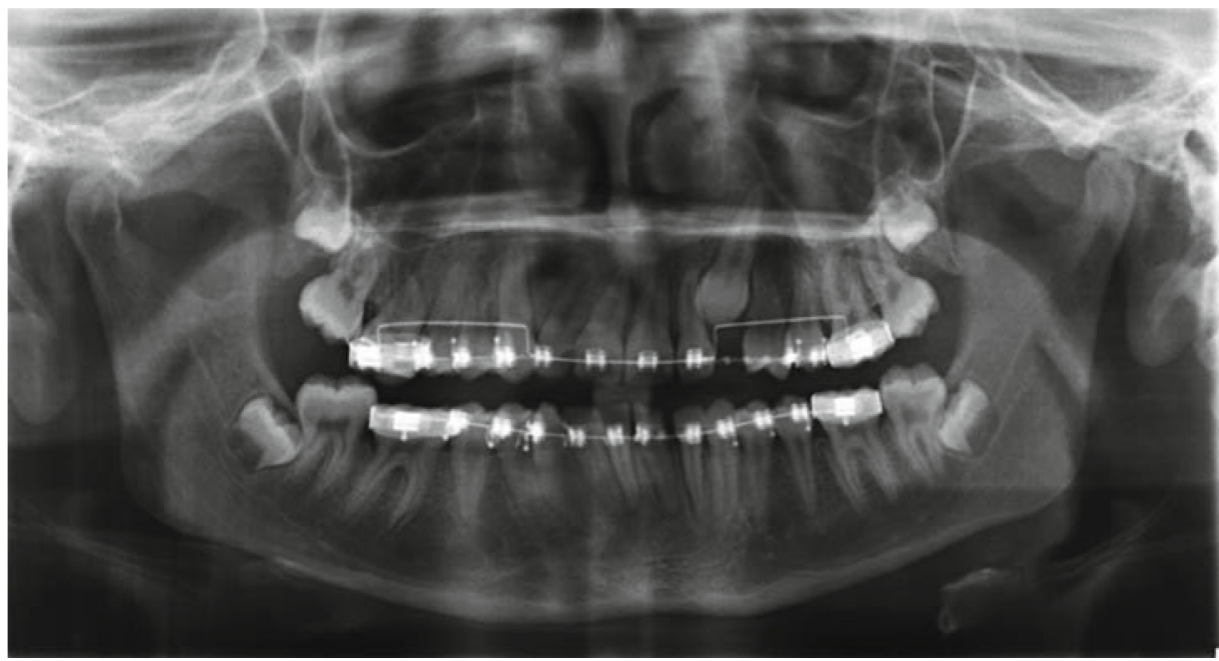

Figure 13

Panoramic radiograph after 18 months. 


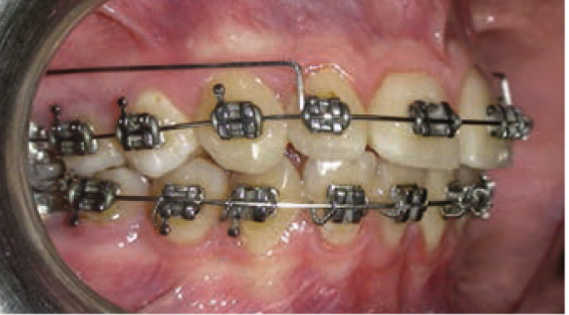

a

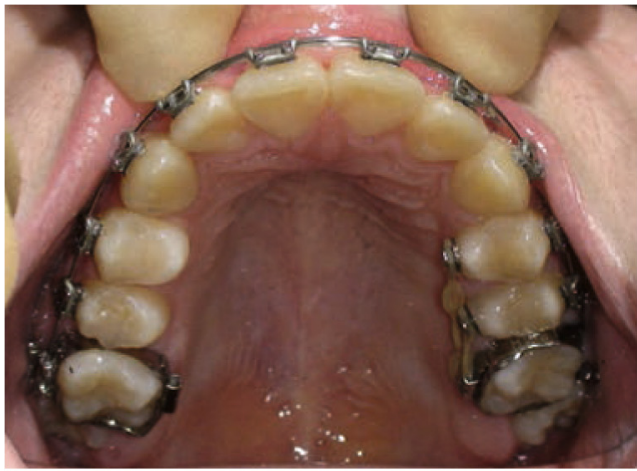

a

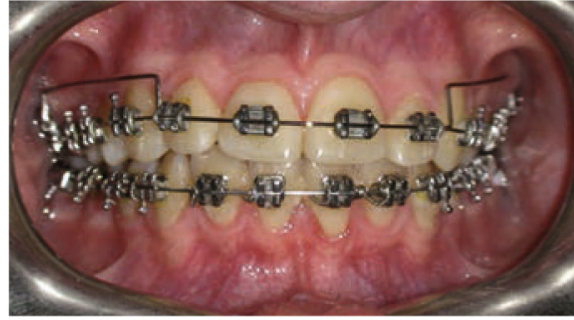

b

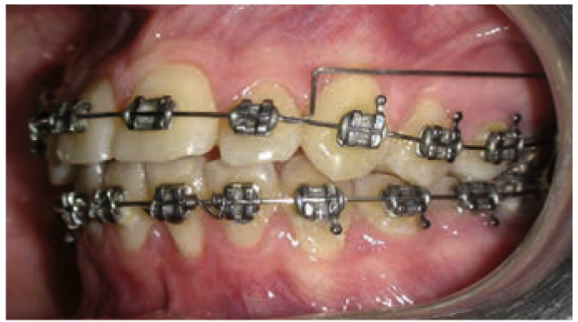

C

Figure 14

$a, b$ and $c$ : Intraorals after 24 months.

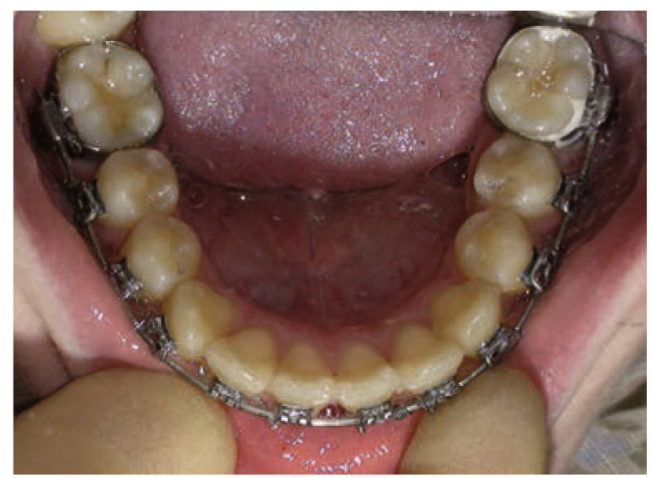

b

Figure 15

a and b: Maxillary and mandibular arches after 24 months.

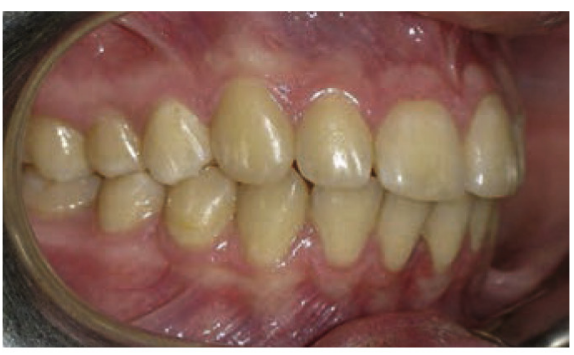

a

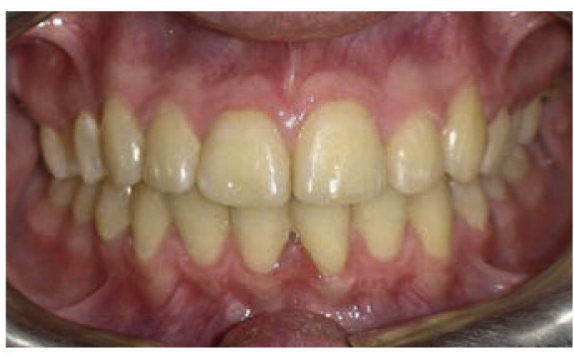

Figure 16

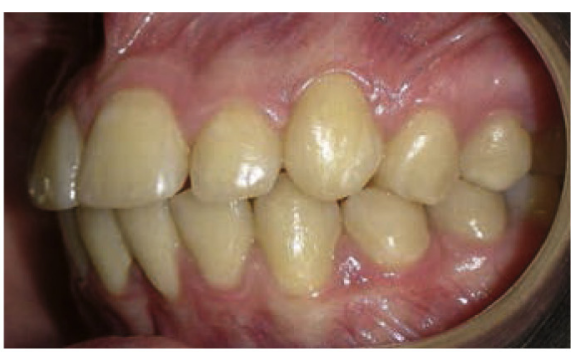

C b

of the end of treatment 30 months. 


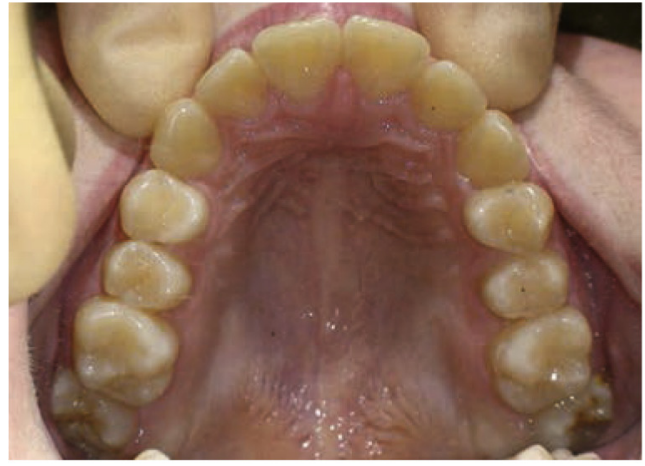

a

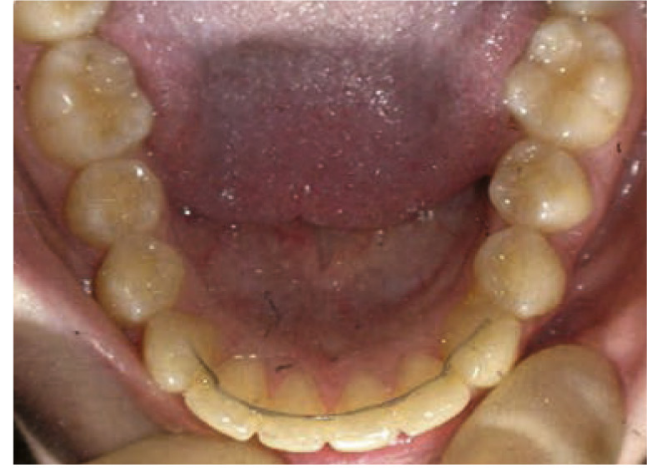

b

Figure 17

$a$ and b: Maxillary and mandibular arches at the end of treatment after 30 months.

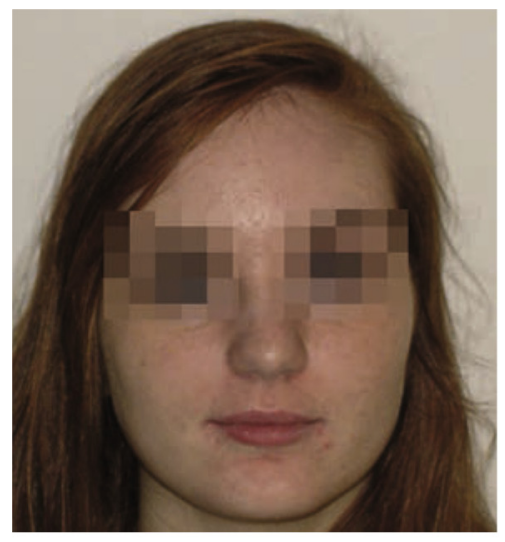

a

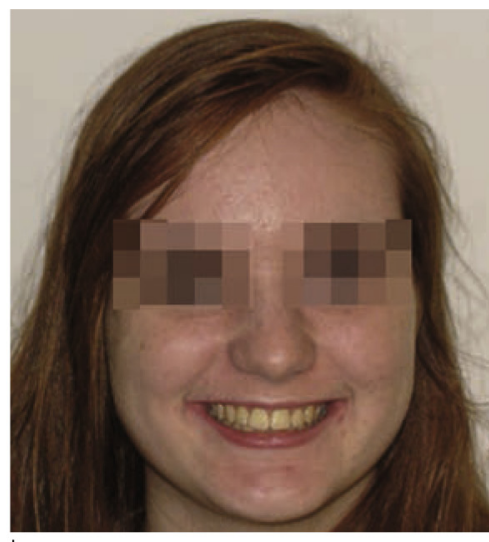

b

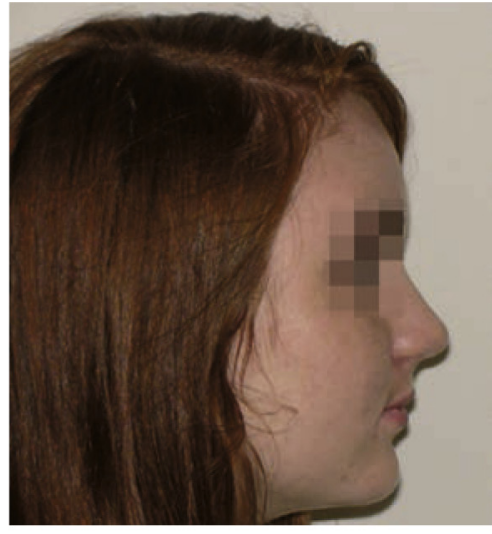

C

Figure 18

a) Frontal face at the end of treatment; b) smiling face at the end of treatment; c) facial profile at the end of treatment.

September 2012 check radiographs (Fig. 19a, b, Fig. 20a, b).

\section{RESULTS OF TREATMENT}

The Class I relationships are firmly in place for both the molars and canines. The incisor crowding was corrected with adequate incisive
5 academic semesters and a year of retention were requested.

relationship: midlines coincident, overjet $2 \mathrm{~mm}$ and overbite $20 \%$.

The esthetic result is very good with a beautiful exposure of the incisors 


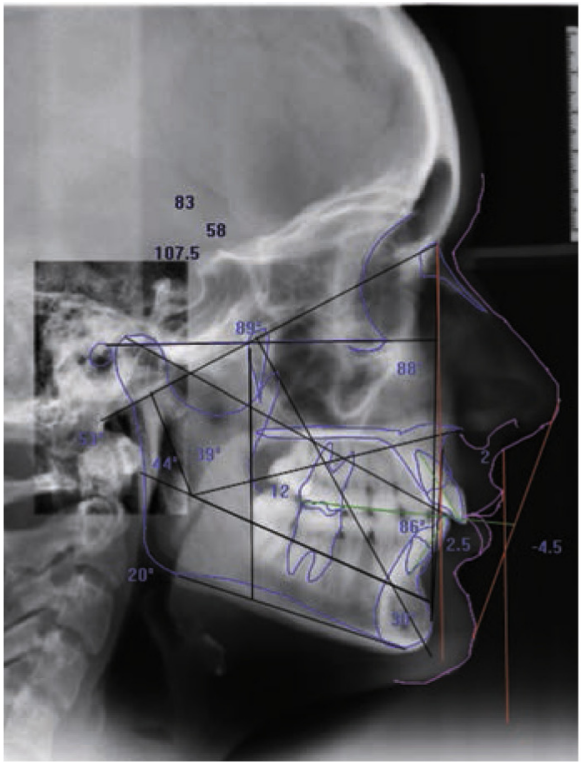

a

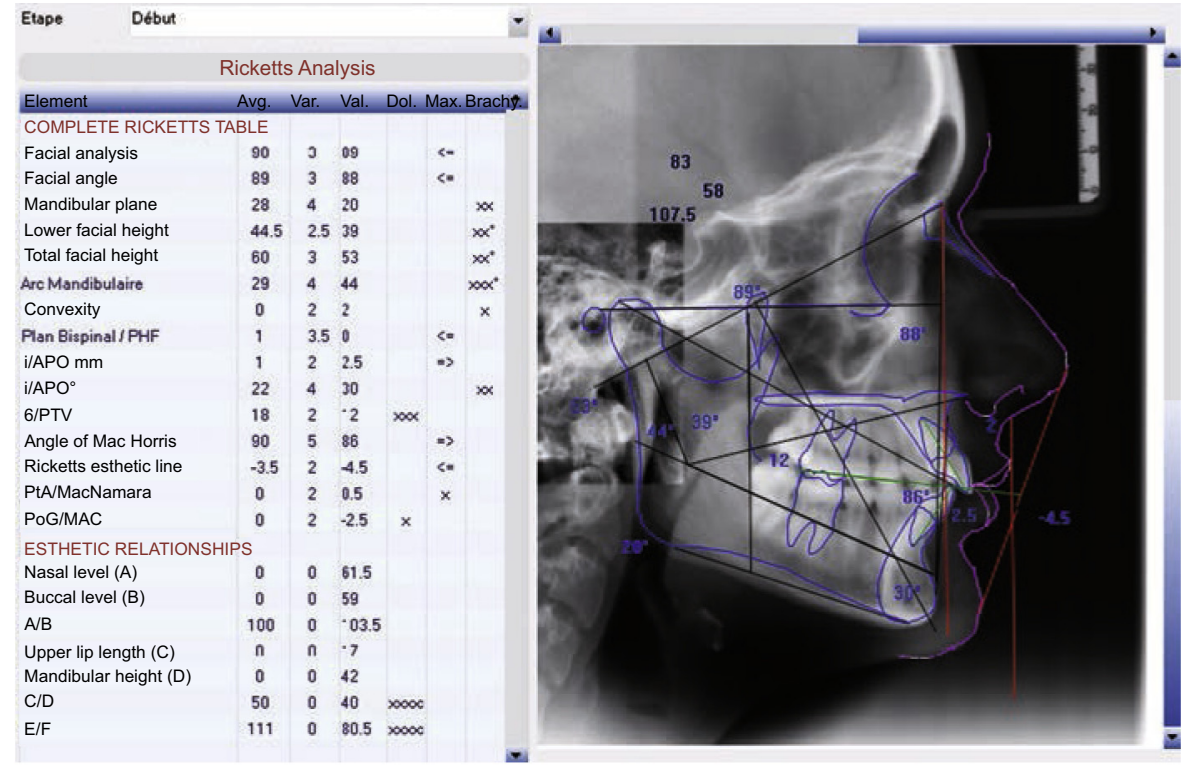

Figure 19

a) End treatment profile teleradiograph 30 months; b) end treatment Ricketts cephalometic analysis.

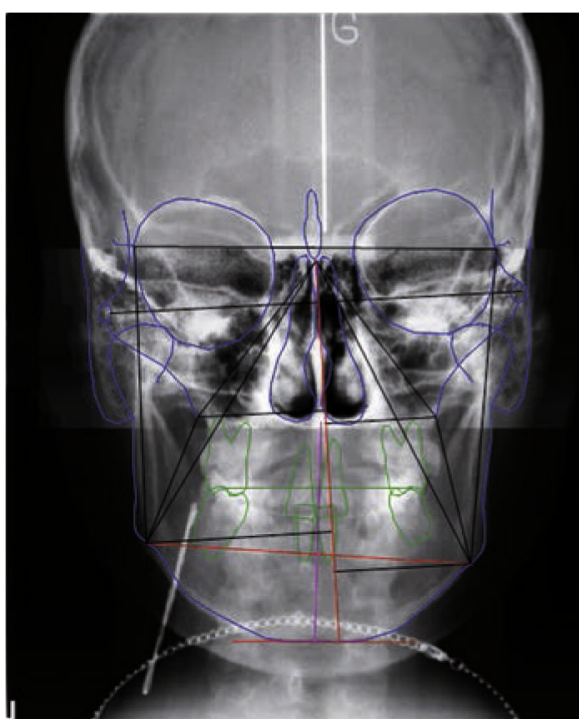

a

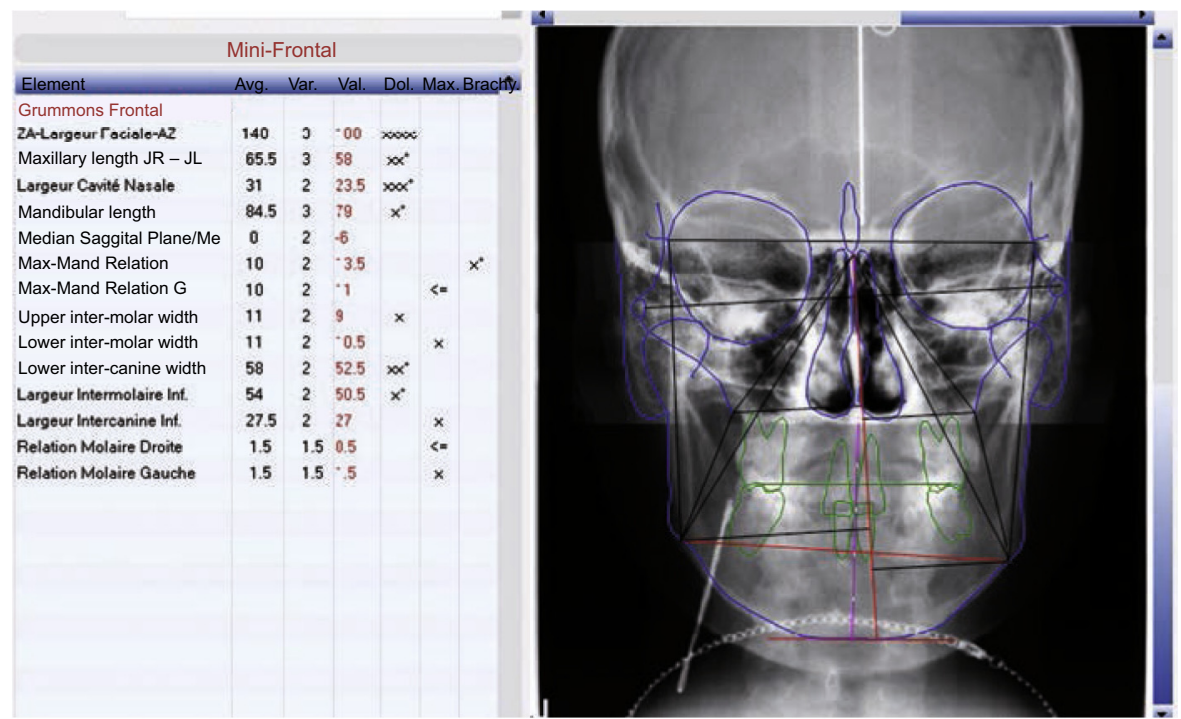

Figure 20

a) End treatment frontal teleradiograph 30 months; b) end treatment Grummons facial analysis. 
while smiling and with full lips thanks to incisor torque that was adapted to the patient's facial type (Fig. 21a, b, Fig. 22a, b).

The global superimposition (begin/ end) images show the torque that was obtained (Fig. 23).
Panoramic of the end of treatment (Fig. 24).

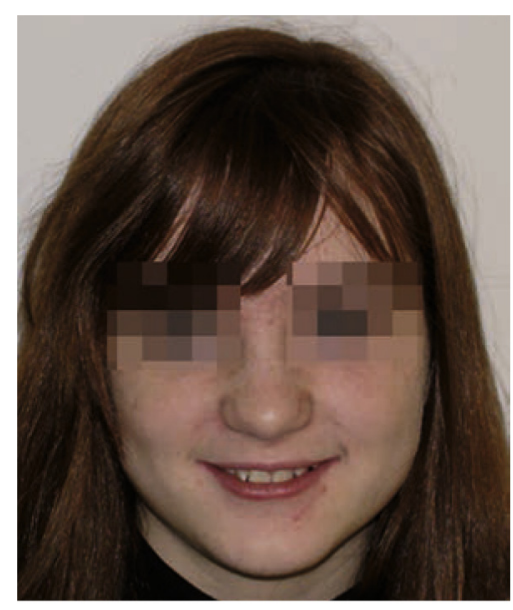

a

Figure 21

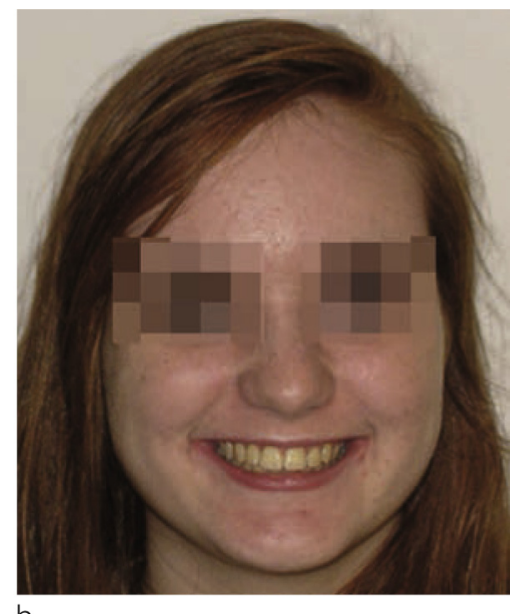

b

a) Begin treatment smile; b) end treatment smile.

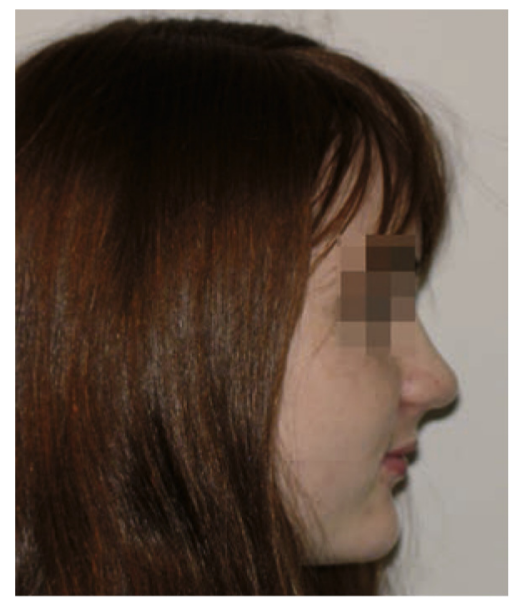

a

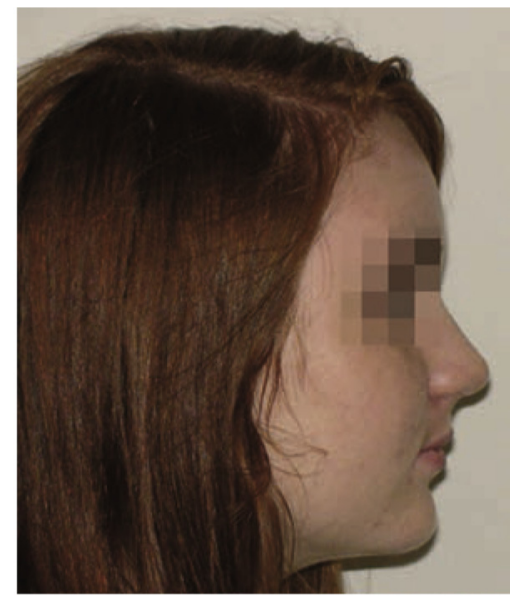

b

Figure 22

a) Begin treatment facial profile; b) end treatment facial profile. 


\section{P. GUEZENEC}

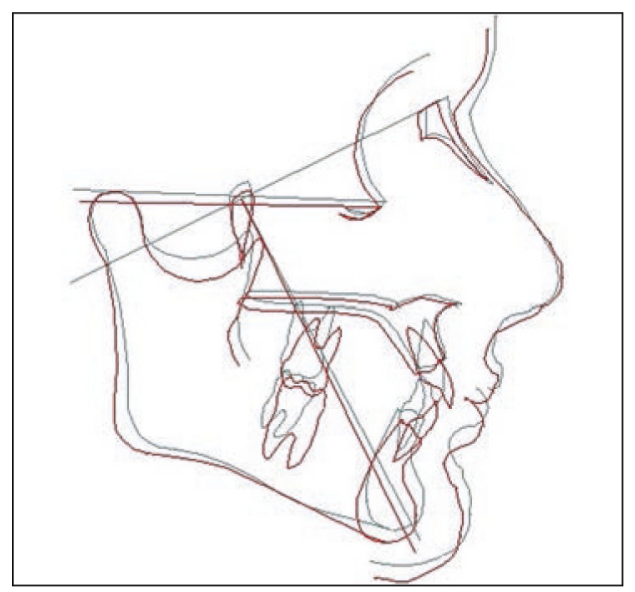

Figure 23

Global superimposition begin/end.

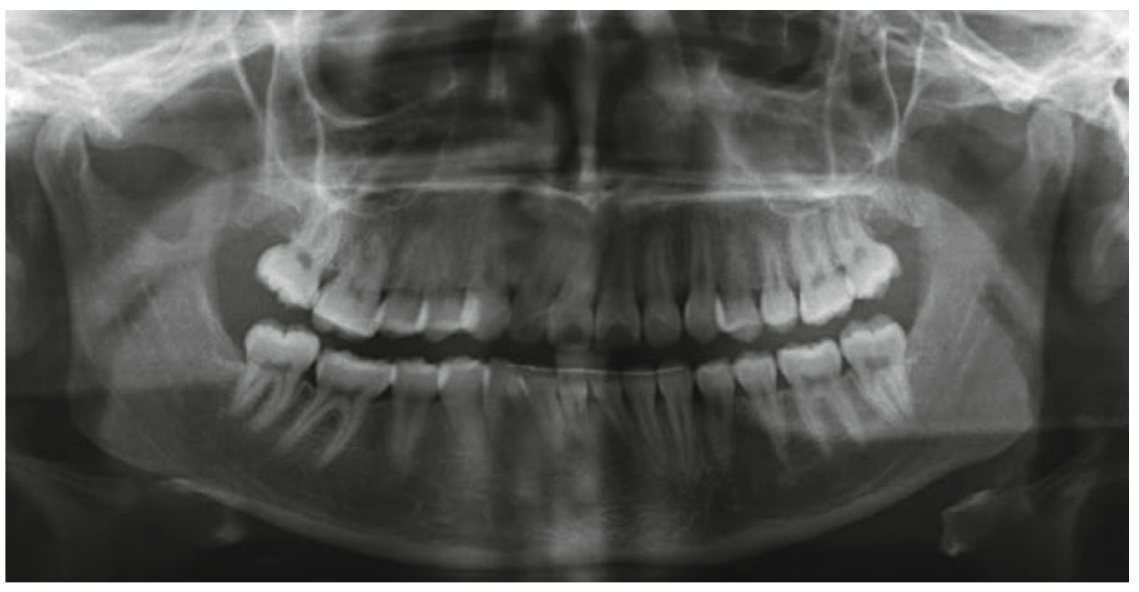

Figure 24

Panoramic radiograph end of treatment.

Conflicts of interest: The author declares no conflict of interest. 